\title{
FATORES ASSOCIADOS À CARGA DE TRABALHO DE ENFERMAGEM EM UNIDADE DE TERAPIA INTENSIVA: REVISÃO INTEGRATIVA
}

\author{
FACTORS ASSOCIATED WITH NURSING WORKLOAD IN THE \\ INTENSIVE CARE UNITS: INTEGRATIVE REVIEW
}

\author{
FACTORES ASOCIADOS CON LA CARGA DE TRABAJO DE \\ ENFERMERÍA EN LA UNIDAD DE CUIDADOS INTENSIVOS: \\ REVISIÓN INTEGRADORA
}

\author{
Mayra Gonçalves Menegueti* \\ Thamiris Ricci de Araújo** \\ Tatiana do Altíssimo Nogueira ${ }^{* * *}$ \\ Francine SancheZ Gulin ${ }^{* * *}$ \\ Ana Maria LaUs ${ }^{* * * *}$
}

\begin{abstract}
RESUMO
Objetivo: Identificar as evidências disponíveis na literatura nacional e internacional sobre fatores associados à carga de trabalho de enfermagem em Unidade de Terapia Intensiva. Método: Trata-se de uma Revisão Integrativa da Literatura realizada no período de janeiro de 2003 a dezembro de 2013 . O levantamento dos estudos foi realizado nas bases de dados Literatura Latino-Americana e do Caribe em Ciências da Saúde e Medical Literature Analysis and Retrieval System Online. Resultados: A amostra final consistiu de 16 artigos. A análise evidenciou que as variáveis que mais foram citadas como preditoras de maior carga de trabalho em Unidade de Terapia Intensiva foram o tempo de permanência na unidade, gravidade e desfecho. A idade do paciente não teve impacto na carga de trabalho na maioria dos estudos. Conclusão: Esta revisão possibilitou conhecer as variáveis que tem sido objeto de investigação enquanto influência sobre carga de trabalho em Unidade de Terapia Intensiva sem, entretanto possibilitar que se afirme tal relação, necessitando novos estudos.
\end{abstract}

Palavras chave: Carga de trabalho, enfermagem, unidade de terapia intensiva.

\footnotetext{
ABSTRACT

Objective: This study aimed to evaluate the evidence available in the national and international literature on the factors associated with nursing workload in the Intensive Care Unit. Method: This is an Integrative Literature Review conducted from January 2003 to December 2013. The survey of studies was conducted on the basis of

*Doutoranda da Escola de Enfermagem de Ribeirão Preto da Universidade de São Paulo, Brasil. Email: mayramenegueti@ usp.br

** Doutoranda da Escola de Enfermagem de Ribeirão Preto da Universidade de São Paulo, Brasil. Email: thamirisricci@ yahoo.com.br

${ }^{* * *}$ Mestranda da Escola de Enfermagem de Ribeirão Preto da Universidade de São Paulo, Brasil. Email: tatiananogueira@ yahoo.com.br

${ }_{* * * *}$ Mestranda da Escola de Enfermagem de Ribeirão Preto da Universidade de São Paulo, Brasil. Email: fran_gulin@hotmail. com

${ }^{* * * *}$ Professora Associada da Escola de Enfermagem de Ribeirão Preto da Universidade de São Paulo, Brasil. Email: analaus@ eerp.usp.br
} 
Latin American and Caribbean Literature Data on Health Sciences and Medical Literature Analysis and Retrieval System Online. Results: The final sample consisted of 16 items. The analysis of these showed that the variables that were mosly cited as predictors of increased workload in the Intensive Care Unit were the length of stay in the unit, severity and outcome. Patient age had no impact on the workload in most studies. Conclusion: This review has helped understand the variables that have received attention as influence on nursing workload in the Intensive Care Unit, although such relationship cannot be confirmed therefore further studies are needed.

Key words: Workload, nursing, intensive care units.

\section{RESUMEN}

Objetivo: Identificar la evidencia disponible en la literatura nacional e internacional sobre los factores asociados con la carga de trabajo de enfermería en Unidades de Cuidados Intensivos. Método: Se trata de una revisión integradora de la literatura realizada en el período enero de 2003 a diciembre de 2013. La búsqueda de estudios se realizó en las bases de datos de Literatura Latinoamericana y del Caribe en Ciencias de la Salud y en Medical Literature Analysis and Retrieval System Online. Resultados: La muestra final estuvo compuesta por 16 artículos. El análisis mostró que las variables que más se citaron como predictoras de mayor carga de trabajo en las Unidades de Cuidados Intensivos fueron la duración de la estadía en la unidad, la gravedad y el resultado. La edad del paciente no tuvo impacto en la carga de trabajo en la mayoría de los estudios. Conclusión: La revisión permitió conocer las variables que han sido objeto de investigación en relación a la influencia en la carga de trabajo en Unidades de Cuidados Intensivos, pero sin permitir afirmar esta relación, lo que requiere estudios adicionales.

Palabras clave: Carga de trabajo, enfermería, unidades de cuidados intensivos.

Fecha recepción: 17/09/15 Fecha aceptación: 30/12/16

\section{INTRODUÇÃO}

A evolução tecnológica na Saúde atinge principalmente unidades de alta complexidade como as Unidades de Terapia Intensiva (UTI) e tem influenciado na mudança do perfil dos pacientes internados, que são altamente dependentes, demandam elevada carga de trabalho de enfermagem, cuidados complexos, estão sujeitos a constantes alterações hemodinâmicas e iminente risco de morte (1).

Este panorama tem chamado a atenção para a qualidade e segurança do paciente nestas unidades. O número inadequado de profissionais de enfermagem tende a determinar a queda da eficácia e qualidade assistencial, prolongando a internação e aumentando os custos do tratamento (2). A redução da equipe de profissionais de enfermagem na tenta- tiva de contenção dos custos, na maioria das vezes, ocorre sem planejamento, provocando impactos no cuidado ao paciente (3). Além disso, uma equipe de enfermagem reduzida ocasiona sobrecarga de trabalho, o que é um fator restritivo ao trabalho multiprofissional (4).

Assim, para um melhor planejamento, alocação, distribuição e controle do quadro de profissionais, torna-se fundamental importância que a gerência de enfermagem avalie a carga de sua equipe numa dada unidade assistencial (5).

A carga média de trabalho da unidade é a soma do produto da quantidade média diária de pacientes assistidos segundo o tipo de cuidado requerido, pelo tempo médio de assistência de enfermagem utilizada, por paciente, de acordo com o grau de dependência (6). 
O conhecimento e a mensuração da carga de trabalho de enfermagem é um dos indicadores para realização do dimensionamento do pessoal de enfermagem, em razão de se constituir uma variável importante para sua realização. A identificação dos fatores associados à carga de trabalho pode fornecer pistas valiosas para a determinação das necessidades de pessoal, bem como para a gestão de cuidados de enfermagem em terapia intensiva (7).

Embora alguns autores (8) indiquem que a adição de variáveis clínicas dos pacientes à medida de carga de trabalho podem contribuir para melhorar as previsões de recursos humanos de enfermagem, não há clareza quanto as variáveis de consenso a serem consideradas nesse processo.

Este estudo teve por objetivo identificar as evidências disponíveis na literatura nacional e internacional sobre os fatores associados à carga de trabalho de enfermagem em UTI.

\section{MÉTODO}

Trata-se de uma Revisão Integrativa (RI) da Literatura. Este método pode tornar os resultados de pesquisas mais acessíveis, reduzindo alguns obstáculos da utilização do conhecimento científico, ao possibilitar ao leitor ter acesso a diversas pesquisas realizadas em um único estudo (9).

A questão norteadora desta RI foi: "Quais fatores podem ser associados à carga de trabalho de enfermagem em UTI?".

O levantamento dos estudos foi realizado nos meses de janeiro e fevereiro de 2014, nas bases de dados Literatura Latino-Americana e do Caribe em Ciências da Saúde (LILACS) e Medical Literature Analysis and Retrieval System Online (MEDLINE) com a combinação dos descritores: carga de trabalho; AND unidades de terapia intensiva (LILACS) e workload AND nursing AND intensive (MEDLINE).
Os critérios de inclusão estabelecidos nesta seleção foram artigos que tivessem por objetivo relatar fatores associados à carga de trabalho de enfermagem em UTI, disponíveis na íntegra, nos idiomas português e inglês, sendo estudos primários e publicados no período de janeiro de 2003 a dezembro de 2013. Os critérios de exclusão aplicados foram: revisões de literatura por serem estudos secundários, estudos que não atendessem ao objetivo do estudo, além de artigos não disponíveis na íntegra.

A primeira seleção dos artigos foi feita pela leitura do título seguida da análise dos resumos por dois dos pesquisadores independentemente, sendo que no caso de divergência na inclusão do artigo, o mesmo foi lido na íntegra e um terceiro pesquisador revia a inclusão do artigo. Quanto aos artigos elegíveis, os mesmos foram lidos na íntegra por dois pesquisadores novamente, de forma independente e extraíram os dados previamente definidos.

Para a coleta de dados foi utilizado um instrumento, construído pelos próprios autores baseado em estudos de revisão disponíveis na literatura, que permitiu: 1) a identificação das publicações (título do artigo e do periódico, identificação do autor principal [instituição de origem e formação profissional], ano de publicação, e locais do estudo); 2) fatores associados e não associados à carga de trabalho de enfermagem; 3) características metodológicas (objetivos do estudo, resultados, limitações e conclusões).

\section{RESULTADOS}

A amostra final consistiu de 16 artigos, sendo que Figura 1 elucida o processo de inclusão, segundo recomendação do Preferred Reporting Items for Systematic Reviews and Meta-Analyses (PRISMA) flowchart (10).

A carga de trabalho em UTI é um assunto em evidência e alguns periódicos têm dado 
Figura 1. Processo de inclusão dos artigos da revisão integrativa sobre carga de trabalho em unidade de terapia intensiva.

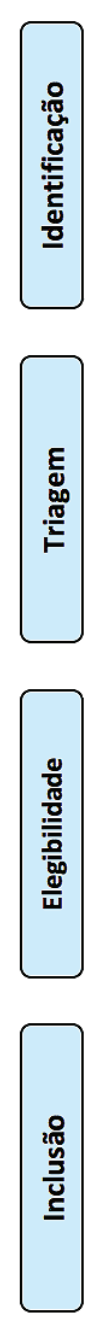

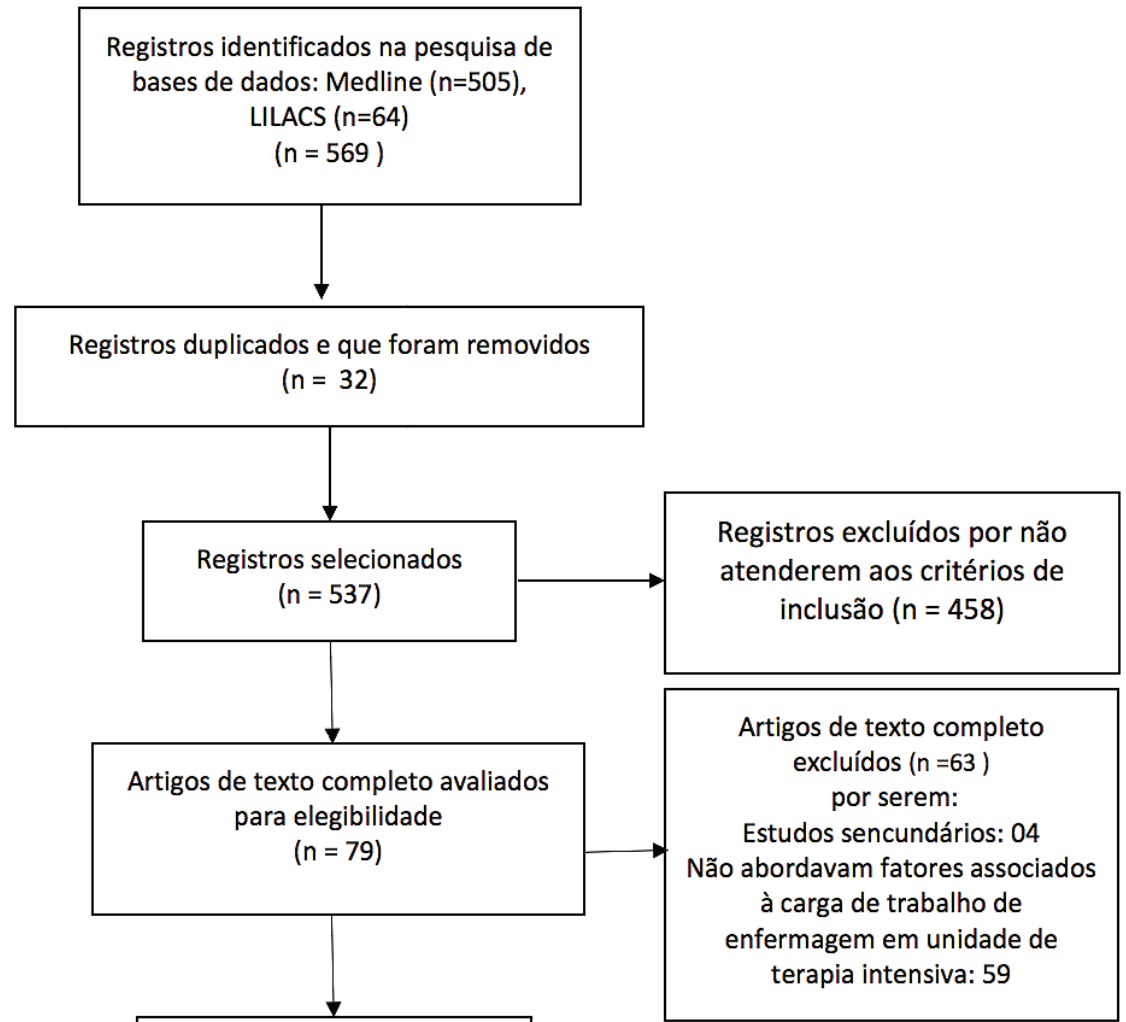

Estudos incluídos na síntese qualitativa $(n=16)$

Fonte: Adaptação do Flow Diagrama do processo de seleção de artigos da revisão integrativa, de acordo com o Preferred Reporting Items for Systematic Reviews and Meta-Analyses (PRISMA).

importância a esta temática, visto que a enfermagem vem argumentando de forma mais fundamentada que a inadequação numérica e qualitativa de recursos pode comprometer a assistência e segurança do paciente. Somase a este aspecto a questão relativa à Lei do Exercício Profissional de Enfermagem (11), que estabelece como atividade privativa do enfermeiro o cuidado ao paciente grave e que pode ser compreendido como um determinante na responsabilização pela assistência prestada.

Os principais resultados encontrados nesta revisão seguem descritos no Quadro 1. 
Quadro 1. Síntese dos estudos primários segundo autores, ano, objetivo, método, amostra e resultados (2003-2013) - Ribeirão Preto, SP, Brasil, 2014.

\begin{tabular}{|c|c|c|c|c|}
\hline Referência & Objetivo & Método & Amostra & $\begin{array}{l}\text { Resultados fatores que } \\
\text { interferem na carga de } \\
\text { trabalho }\end{array}$ \\
\hline
\end{tabular}

\begin{tabular}{lllll}
\hline $\begin{array}{l}\text { Ducci, Padilha, } \\
\text { Telles, Gutierrez } \\
\text { (12) }\end{array}$ & $\begin{array}{l}\text { Verificar a associação } \\
\text { das variáveis: } \\
\text { procedência, tempo } \\
\text { de permanência e } \\
\text { condições de alta da }\end{array}$ & $\begin{array}{l}\text { Estudo descritivo- } \\
\text { exploratório, } \\
\text { retrospectivo e } \\
\text { UTI com a gravidade }\end{array}$ & $\begin{array}{l}\text { Amostra } \\
\text { composta } \\
\text { por 77 }\end{array}$ & $\begin{array}{l}\text { Procedência (maior TISS } \\
\text { da semi intensiva do } \\
\text { que do pronto Socorro). }\end{array}$ \\
$\begin{array}{l}\text { dos pacientes e } \\
\text { demanda de trabalho } \\
\text { de enfermagem pelo }\end{array}$ & & $\begin{array}{l}\text { Tempo de permanência } \\
\text { (maior tempo maior }\end{array}$ \\
& & & $\begin{array}{l}\text { carga). Desfecho (pacientes } \\
\text { que foram a óbito maior } \\
\text { carga) }\end{array}$ \\
\end{tabular}

TISS-28

\begin{tabular}{|c|c|c|c|c|}
\hline $\begin{array}{l}\text { Garcia, } \\
\text { Gonçalves, } \\
\text { Ducci, Toffoleto, } \\
\text { Ribeiro, Padilha } \\
\text { (13) }\end{array}$ & $\begin{array}{l}\text { Identificar a } \\
\text { prevalência dos } \\
\text { itens componentes } \\
\text { das categorias } \\
\text { de intervenções } \\
\text { terapêuticas } \\
\text { realizadas na UTI, } \\
\text { segundo o TISS-28 }\end{array}$ & $\begin{array}{l}\text { Estudo descritivo, } \\
\text { retrospectivo, } \\
\text { longitudinal }\end{array}$ & 77 pacientes & $\begin{array}{l}\text { Procedência (maior TISS } \\
\text { da semi intensiva do } \\
\text { que do pronto Socorro). } \\
\text { Tempo de permanência } \\
\text { (maior tempo maior } \\
\text { carga). Desfecho (pacientes } \\
\text { que foram a óbito maior } \\
\text { carga) }\end{array}$ \\
\hline $\begin{array}{l}\text { Ciampone, } \\
\text { Gonçalves, Maia, } \\
\text { Padilha (14) }\end{array}$ & $\begin{array}{l}\text { Comparar as } \\
\text { necessidades } \\
\text { de cuidado de } \\
\text { enfermagem e } \\
\text { intervenções } \\
\text { terapêuticas de } \\
\text { pacientes idosos e } \\
\text { não idosos em UTI } \\
\text { segundo o NAS }\end{array}$ & $\begin{array}{l}\text { Estudo } \\
\text { quantitativo }\end{array}$ & $\begin{array}{l}50 \text { pacientes } \\
\text { sendo } \\
\text { otidas } 339 \\
\text { medidas do } \\
\text { escore NAS }\end{array}$ & Nenhum \\
\hline $\begin{array}{l}\text { Balsanelli, Zanei, } \\
\text { Whitaker (15) }\end{array}$ & $\begin{array}{l}\text { Verificar a relação } \\
\text { entre a carga } \\
\text { de trabalho de } \\
\text { enfermagem (TISS- } \\
\text { 28) e as variáveis: } \\
\text { gravidade da doença } \\
\text { (APACHE II), } \\
\text { sobrevida e, o tempo } \\
\text { de permanência na } \\
\text { UTI dos pacientes } \\
\text { cirúrgicos. }\end{array}$ & $\begin{array}{l}\text { Estudo quantitativo } \\
\text { e retrospectivo }\end{array}$ & $\begin{array}{l}143 \\
\text { pacientes } \\
\text { cirúrgicos } \\
\text { admitidos } \\
\text { em três UTI }\end{array}$ & $\begin{array}{l}\text { Pacientes de maior } \\
\text { gravidade, não } \\
\text { sobreviventes e com maior } \\
\text { tempo de permanência } \\
\text { na UTI tiveram maiores } \\
\text { valores do NAS }\end{array}$ \\
\hline
\end{tabular}


Continuação Quadro 1.

\begin{tabular}{|c|c|c|c|c|}
\hline $\begin{array}{l}\text { Gonçalves, } \\
\text { Garcia, Toffoleto, } \\
\text { Telles, Padilha } \\
\text { (16) }\end{array}$ & $\begin{array}{l}\text { Descrever a demanda } \\
\text { de trabalho de } \\
\text { enfermagem } \\
\text { segundo o NAS e } \\
\text { verificar a influência } \\
\text { das variáveis: } \\
\text { idade, tempo de } \\
\text { permanência, } \\
\text { procedência e destino } \\
\text { após a saída da } \\
\text { unidade na carga } \\
\text { de trabalho de } \\
\text { enfermagem }\end{array}$ & Estudo quantitativo & $\begin{array}{l}\text { População } \\
\text { constituída } \\
\text { por } 50 \\
\text { pacientes } \\
\text { com idade } \\
\text { igual ou } \\
\text { maior do } \\
\text { que } 18 \text { anos }\end{array}$ & $\begin{array}{l}\text { Pacientes que foram a } \\
\text { óbito e ficaram mais dias } \\
\text { internados tiveram NAS } \\
\text { superior }\end{array}$ \\
\hline $\begin{array}{l}\text { Padilha, Sousa, } \\
\text { Kimura, } \\
\text { Miyadahira, } \\
\text { Dacruz, Vattimo, } \\
\text { et al. (17) }\end{array}$ & $\begin{array}{l}\text { Caracterizar a } \\
\text { gravidade dos } \\
\text { pacientes e a } \\
\text { carga de trabalho } \\
\text { de enfermagem } \\
\text { utilizando o TISS-28 }\end{array}$ & Estudo prospectivo & $\begin{array}{l}271 \\
\text { pacientes } \\
\text { em } 11 \text { UTI }\end{array}$ & $\begin{array}{l}\text { Maior TISS nos pacientes } \\
\text { que foram a óbito e que } \\
\text { tinham maior gravidade, } \\
\text { bem como aqueles } \\
\text { provenientes da UTI de } \\
\text { pacientes transplantados } \\
\text { hepáticos }\end{array}$ \\
\hline $\begin{array}{l}\text { Kiekkas, } \\
\text { Brokalaki, } \\
\text { Manolis, Samios, } \\
\text { Skartsani, } \\
\text { Baltopoulos (18) }\end{array}$ & $\begin{array}{l}\text { Investigar se a } \\
\text { gravidade dos } \\
\text { pacientes de UTI } \\
\text { pode ser usada } \\
\text { para a previsão da } \\
\text { carga de trabalho de } \\
\text { enfermagem }\end{array}$ & $\begin{array}{l}\text { Estudo prospectivo } \\
\text { quantitativo }\end{array}$ & $\begin{array}{l}138 \\
\text { pacientes }\end{array}$ & $\begin{array}{l}\text { APACHE II é um } \\
\text { importante indicador } \\
\text { precoce de carga de } \\
\text { trabalho de enfermagem }\end{array}$ \\
\hline $\begin{array}{l}\text { Gonçalves, } \\
\text { Padilha (19) }\end{array}$ & $\begin{array}{l}\text { Analisar a carga } \\
\text { de trabalho de } \\
\text { enfermagem e } \\
\text { identificar os fatores } \\
\text { associados a ela } \\
\text { no primeiro dia } \\
\text { de internação dos } \\
\text { pacientes na UTI }\end{array}$ & $\begin{array}{l}\text { Estudo de corte } \\
\text { transversal e } \\
\text { retrospectivo }\end{array}$ & $\begin{array}{l}\text { Cinco UTI. } \\
\text { Amostra } \\
\text { de } 214 \\
\text { pacientes } \\
\text { (banco de } \\
\text { dados) }\end{array}$ & $\begin{array}{l}\text { Tempo de permanência } \\
\text { como fator preditor de } \\
\text { elevada carga de trabalho }\end{array}$ \\
\hline $\begin{array}{l}\text { Padilha, Sousa, } \\
\text { Queijo, Mendes, } \\
\text { Miranda ( } 8 \text { ) }\end{array}$ & $\begin{array}{l}\text { Explorar a associação } \\
\text { do NAS com } \\
\text { variáveis como sexo, } \\
\text { idade, tempo de } \\
\text { permanência, alta da } \\
\text { UTI, TISS-28 e SAPS } \\
\text { II }\end{array}$ & $\begin{array}{l}\text { Estudo prospectivo, } \\
\text { descritivo, } \\
\text { exploratório }\end{array}$ & $\begin{array}{l}200 \\
\text { pacientes, } \\
\text { sendo } 144 \\
\text { de UTI } \\
\text { geral e } 56 \\
\text { de UTI } \\
\text { neurológica }\end{array}$ & $\begin{array}{l}\text { Tempo de permanência na } \\
\text { UTI (univariada) } \\
\text { Desfecho (maior carga } \\
\text { dos pacientes que foram a } \\
\text { óbito) }\end{array}$ \\
\hline
\end{tabular}


Continuação Quadro 1.

\begin{tabular}{llll}
\hline Kiekkas, & Identificar se os & Estudo prospectivo & 361 \\
Sakellaropoulos, & pacientes com febre & e descritivo & pacientes \\
Brokalaki, & na UTI geral & \\
Manolis, Samios, & demandam maior & \\
Skartsani, et al. & $\begin{array}{l}\text { carga de trabalho de } \\
\text { enfermagem }\end{array}$ & \\
$(20)$ & &
\end{tabular}

Foram 188 pacientes febris, sendo que estes eram mais jovens, tiveram APACHE II mais altos, maior duração da ventilação mecânica e permanecem mais tempo na UTI. A média diária do TISS-28 foram maiores para pacientes febris do que para pacientes afebris

Sousa, Gonçalves, Descrever a carga Toffoleto, Leão, de trabalho de Padilha (21) enfermagem entre indivíduos idosos, comparar a demanda de trabalho, a gravidade e o risco de mortalidade entre idosos e identificar preditores de alta demanda de trabalho de enfermagem

\section{Estudo} epidemiológico de corte transversal

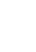

\begin{tabular}{|c|c|c|c|c|}
\hline $\begin{array}{l}\text { Sousa, Padilha, } \\
\text { Nogueira, } \\
\text { Miyadahira, } \\
\text { Oliveira (22) }\end{array}$ & $\begin{array}{l}\text { Comparar a } \\
\text { carga de trabalho } \\
\text { de enfermagem } \\
\text { requerida por } \\
\text { pacientes adultos, } \\
\text { idosos e muito idosos } \\
\text { na admissão e alta da } \\
\text { UTI }\end{array}$ & $\begin{array}{l}\text { Estudo prospectivo, } \\
\text { longitudinal }\end{array}$ & $\begin{array}{l}\text { Casuística } \\
\text { composta } \\
\text { de } 600 \\
\text { pacientes }\end{array}$ & $\begin{array}{l}\text { Os pacientes com idade } \\
\text { mais avançada mantiveram } \\
\text { demanda mais elevada } \\
\text { de cuidados do que os } \\
\text { adultos, após a alta }\end{array}$ \\
\hline $\begin{array}{l}\text { Inoue, Kuroda, } \\
\text { Matsuda ( } 23)\end{array}$ & $\begin{array}{l}\text { Verificar quais } \\
\text { variáveis } \\
\text { demográficas e } \\
\text { clínicas de pacientes } \\
\text { que influenciam } \\
\text { diretamente a carga } \\
\text { de trabalho } \\
\text { da enfermagem }\end{array}$ & $\begin{array}{l}\text { Estudo descritivo } \\
\text { exploratório }\end{array}$ & $\begin{array}{l}\text { População } \\
\text { constituiu- } \\
\text { se de } \\
100 \\
\text { pacientes }\end{array}$ & $\begin{array}{l}\text { Pacientes cirúrgicos } \\
\text { demandaram maior carga } \\
\text { de trabalho }\end{array}$ \\
\hline $\begin{array}{l}\text { Coelho, Queijo, } \\
\text { Andolhe, } \\
\text { Gonçalves, } \\
\text { Padilha (24) }\end{array}$ & $\begin{array}{l}\text { Verificar a associação } \\
\text { entre a carga } \\
\text { de trabalho e as } \\
\text { variáveis: idade, } \\
\text { sexo, procedência, } \\
\text { tipo de tratamento, } \\
\text { gravidade, } \\
\text { disfunção orgânica, } \\
\text { tempo de internação } \\
\text { na UTI e mortalidade } \\
\text { na unidade }\end{array}$ & Estudo quantitativo & $\begin{array}{l}\text { Amostra } \\
\text { constituída } \\
\text { por } 100 \\
\text { pacientes }\end{array}$ & $\begin{array}{l}\text { Os autores relatam que } \\
\text { quase houve associação } \\
\text { entre o sexo masculino } \\
\text { que exigiram maior carga } \\
\text { de trabalho do que o } \\
\text { feminino }\end{array}$ \\
\hline
\end{tabular}


Continuação Quadro 1.

\begin{tabular}{|c|c|c|c|c|}
\hline $\begin{array}{l}\text { Queijo, Martins, } \\
\text { Andolhe, } \\
\text { Oliveira, Barbosa, } \\
\text { Padilha (25) }\end{array}$ & $\begin{array}{l}\text { Avaliar e identificar } \\
\text { os fatores associados } \\
\text { a carga de trabalho de } \\
\text { enfermagem em UTI } \\
\text { neurológica }\end{array}$ & $\begin{array}{l}\text { Estudo descritivo } \\
\text { transversal }\end{array}$ & $\begin{array}{l}100 \\
\text { pacientes }\end{array}$ & $\begin{array}{l}\text { A severidade da doença } \\
\text { (avaliada pelo SAPS II) foi } \\
\text { associada diretamente e } \\
\text { a idade foi inversamente } \\
\text { correlacionada com a carga } \\
\text { de trabalho de enfermagem }\end{array}$ \\
\hline $\begin{array}{l}\text { Carmona-Monge, } \\
\text { Jara-Pérez, } \\
\text { Quirós-Herranz, } \\
\text { Rollán- } \\
\text { Rodríguez, } \\
\text { Cerrillo } \\
\text { González, García- } \\
\text { Gómez, et al. (26) }\end{array}$ & $\begin{array}{l}\text { Avaliar a carga de } \\
\text { trabalho na admissão } \\
\text { e alta dos pacientes } \\
\text { na UTI em três } \\
\text { grupos (síndrome } \\
\text { coronária aguda, } \\
\text { insuficiência } \\
\text { respiratória aguda } \\
\text { e sepse) segundo o } \\
\text { NAS }\end{array}$ & $\begin{array}{l}\text { Estudo prospectivo } \\
\text { e descritivo }\end{array}$ & $\begin{array}{l}\text { Durante } \\
27 \text { meses, } \\
\text { foram } \\
\text { incluídos } \\
563 \\
\text { pacientes }\end{array}$ & $\begin{array}{l}\text { Diagnóstico, sendo } \\
\text { maior carga de trabalho } \\
\text { de enfermagem dos } \\
\text { pacientes com insuficiência } \\
\text { respiratória aguda e sepse. } \\
\text { Durante os primeiros sete } \\
\text { dias de internação esta } \\
\text { diferença manteve-se, } \\
\text { desaparecendo ao oitavo } \\
\text { dia. }\end{array}$ \\
\hline
\end{tabular}

A análise dos artigos evidenciou que as variáveis que mais foram citadas como preditoras de maior carga de trabalho em UTI foram o tempo de permanência na unidade, gravidade e desfecho. Os pacientes que foram a óbito e também os de internação mais prolongada em UTI, provavelmente, foram os que apresentavam uma condição mais instável, com necessidade de acompanhamento rigoroso e múltiplas intervenções terapêuticas e, como conseqüência, uma carga de trabalho superior (17).

\section{DISCUSSÃO E CONCLUSÃO}

O paciente grave demanda maior carga de trabalho resultante de um maior tempo gasto em intervenções médicas e de enfermagem, observação e monitoramento dos sinais vitais e resultados laboratoriais (25). Estudo conduzido entre três subgrupos de pacientes: cardiológicos, neurológicos e clínico cirúrgicos gerais, encontrou correlação moderada entre a gravidade e carga de trabalho de enfermagem, apenas no grupo de pacientes neurológicos, o que sinaliza para a necessi- dade de análises mais consistentes quanto as diferentes intervenções a que esses pacientes estão submetidos (27).

Alguns autores questionam se a gravidade influencia o Nursing Activities Score (NAS) apenas na admissão ou durante a internação, uma vez que a maior demanda de cuidado nas $24 \mathrm{~h}$ pode ser atribuída a complexidade do quadro clínico do paciente. Nesse sentido, investigação conduzida em um hospital especializado no atendimento de pacientes em cardiopneumologia, encontrou média NAS nas primeiras $24 \mathrm{~h}$ de $82,4 \%$ e na segunda avaliação em $72 \mathrm{~h}$ valor médio de $58,1 \%$. Tal dado se opõe a alguns estudos nos quais a gravidade do paciente nas $24 \mathrm{~h}$, não influenciou o aumento da carga de trabalho, a qual se mostrou associada ao tempo de internação e às complicações (28).

Entretanto, os resultados de um estudo em uma UTI especializada em trauma que buscou os fatores relacionados a elevada carga de trabalho, identificou sexo, complicações clínicas e múltiplas intervenções como sendo aqueles que se associaram a um NAS elevado (29).

Há de se considerar que a vivência diária dos profissionais de enfermagem em unida- 
des de terapia intensiva provoca uma sensação de que o paciente grave exige maior tempo de assistência, tanto na realização dos procedimentos rotineiros no momento da admissão, como durante sua permanência, em virtude das instabilidades orgânicas que se instalam ao longo de sua permanência nestas unidades (15).

Por outro lado, outros autores discutem que o paciente em estado grave, inconsciente, imóvel e com todas as suas necessidades fisiológicas monitorizadas por suporte tecnológico, quando admitidos na UTI, podem requerer menos tempo de assistência de enfermagem quando comparados com pacientes ansiosos, assustados, estressados com o ambiente e capazes de comunicar dor e desconforto (30).

A idade do paciente, como fator associado à carga de trabalho, não foi relatada em vários estudos como uma variável que tivesse impacto na carga de trabalho $(8,16,19,24)$, contrariando a percepção dos profissionais de enfermagem de que os idosos respondem menos a certas medidas terapêuticas e, portanto, requerem mais cuidados (22).

Ressalta-se que a relevância em identificar previamente os fatores associados à alta carga de trabalho de enfermagem em UTI é um recurso importante no momento da admissão do paciente, com vistas a estimar a carga de trabalho que o mesmo demandará e consequentemente dimensionar os profissionais de enfermagem de modo a garantir a qualidade e eficiência do cuidado prestado (19).

Além disso, para a realização do provimento de quantitativo de pessoal adequado na UTI é exigido que os enfermeiros se apropriem de metodologias para a medida de carga de trabalho de enfermagem e identifiquem os fatores que a influenciam, a fim de serem bem sucedidos nas negociações com os administradores hospitalares.

A revisão dos estudos realizados até o momento possibilitou conhecer as variáveis que tem sido objeto de investigação enquanto influência sobre carga de trabalho de enfer- magem em UTI sem, entretanto possibilitar que se afirme tal relação. No entanto, parece haver uma tendência dos estudos em elencar como preditores de maior carga de trabalho em UTI o tempo de permanência na unidade, gravidade e desfecho. Os estudos de associação exigem o atendimento de alguns pressupostos para sua realização tais como estimar o tamanho da amostra e controle das variáveis de confundimento. Além disso, quando é objetivo da investigação generalizar os resultados encontrados, recomenda-se a realização de estudos multicêntricos. Embora a consistência metodológica não tenha sido alvo de análise desta pesquisa, verificou-se que a maioria dos estudos selecionados não atendeu a estes requisitos na sua totalidade, razão pela qual instiga a necessidade de se realizar novas pesquisas que respondam à questão de investigação.

\section{REFERÊNCIAS}

1. Inoue KC, Matsuda LM. Dimensionamento da equipe de enfermagem da UTI adulto de um hospital ensino. Rev. Eletr. de Enf. [Internet]. 2009 [citado 30 jan de 2014]; 11(1): 55-63. Disponível em: https://www.fen.ufg.br/fen_revista/v11/n1/ v1ln1a07.htm

2. Queijo AF, Padilha KG. Nursing Activities Score (NAS): adaptação transcultural e validação para a língua portuguesa. Rev Esc Enferm USP 2009; 43: 1009-1014.

3. Tallier P. How many nurses are enough? A Pilot study measuring nursing care hours per patient day and patient outcomes. Aquichán. 2007; 7(1): 37-53.

4. Araujo Neto JD, Silva ISP, Zanin LE, Andrade AP, Moraes KM. Profissionais de saúde da unidade de terapia intensiva: percepção dos fatores restritivos da atuação multiprofissional. Rev Bras Promoç Saúde [Internet]. 2016 [citado 30 jan 2017]; 29(1): 43-50. Disponível em: 
file://C:/Users/UFA013315/Downloads/ 4043-19176-1-PB\%20(1).pdf

5. Rogenski KE, Fugulin FMT, Gaidzinski RR, Rogenski NMB. Tempo de assistência de enfermagem em instituição hospitalar de ensino. Rev Esc Enferm USP. 2011; 45(1): 223-9.

6. Gaidzinski RR, Kurcgant P. Dimensionamento de pessoal de enfermagem: vivência de enfermeiras. Nursing. 1998; 2(1): 28-34.

7. Lundgrén-Laine $H$, Suominen T. Nursing intensity and patient classification at an adult intensive care unit (ICU). Intensive Crit Care Nurs. 2007; 23: 97-103.

8. Padilha KG, Sousa RMC, Queijo AF, Mendes AM, Miranda DR. Nursing Activities Score in the intensive care unit: analysis of the related factors. Intensive Crit Care Nurs. 2008; 24: 197-204.

9. Whittemore R, Knalf K. The integrative review: updated methodology. J Adv Nurs. 2005; 52(5): 546-53.

10. Moher D, Liberati A, Tetzlaff J, Altman DG. PRISMA Group. Preferred reporting items for systematic reviews and meta-analyses: the PRISMA statement. PLoS Med [Internet]. 2009 [citado 30 jan 2014]; 6(7): e1000097. Disponível em: https://www.ncbi.nlm.nih.gov/pubmed/19621072

11. Lei $\mathrm{N}^{\circ}$ 7.498. Dispõe sobre a regulamentação do exercício da Enfermagem e dá outras providências. Diário Oficial da República Federativa do Brasil. 26 Jun 1986 [citado 30 jan 2014]. Seção 1. Disponível em http://www.cofen.gov.br/lei-n-74 9886-de-25-de-junho-de-1986_4161. html

12. Ducci AJ, Padilha KG, Telles SCR, Gutierrez BAO. Gravidade de Pacientes e Demanda de Trabalho de Enfermagem em Unidade de Terapia Intensiva: Análise Evolutiva Segundo o TISS-28. Rev Bras Ter Intensiva. 2004; 16(1): 22-27.

13. Garcia PC, Gonçalves LA, Ducci AJ, Toffoleto MC, Ribeiro SC, Padilha KG. In- tervenções terapêuticas em Unidade de Terapia Intensiva: análise segundo o Therapeutic Intervention Scoring System-28 (TISS-28). Rev Bras Enferm. 2005; 58(2): 194-199.

14. Ciampone JT, Gonçalves LA, Maia FOM, Padilha KG. Necessidades de cuidados de enfermagem e intervenções terapêuticas em Unidade de Terapia Intensiva: estudo comparativo entre pacientes idosos e não idosos. Acta Paul Enferm. 2006; 19(1): 28-35.

15. Balsanelli AP, Zanei SSSV, Whitaker IY. Relationships among nursing workload, illness severity, and the survival and length of stay of surgical patients in ICUs. Acta Paul Enferm. 2006; 19(1): 16-20.

16. Gonçalves LA, Garcia PC, Toffoleto MC, Telles SCR, Padilha KG. The need for nursing care in Intensive Care Units: daily patient assessment according to the Nursing Activities Score (NAS). Rev Bras Enferm. 2006; 59(1): 56-60.

17. Padilha K, Sousa R, Kimura M, Miyadahira A, Dacruz D, Vattimo M, et al. Nursing workload in intensive care units: A study using the Therapeutic Intervention Scoring System-28 (TISS-28). Intensive Crit Care Nurs. 2007; 23: 162-169.

18. Kiekkas P, Brokalaki H, Manolis E, Samios A, Skartsani C, Baltopoulos G. Patient severity as an indicator of nursing workload in the intensive care unit. Nurs Crit Care. 2007; 12(1): 34-41.

19. Gonçalves LA, Padilha KG. Fatores associados à carga de trabalho de enfermagem em Unidade de Terapia Intensiva. Rev Esc Enferm USP. 2007; 41(4): 645-52.

20. Kiekkas P, Sakellaropoulos GC, Brokalaki H, Manolis E, Samios A, Skartsani C, et al. Nursing workload associated with fever in the general intensive care unit. Am J Crit Care. 2008; 17(6): 522-31.

21. Sousa CR, Gonçalves LA, Toffoleto MC, Leão K, Padilha KG. Predictors of nursing workload in elderly patients admitted to intensive care units. Rev Lat Am Enfer- 
magem. 2008; 16(2): 218-23.

22. Sousa RMC, Padilha KG, Nogueira LS, Miyadahira AMK, Oliveira VCR. Nursing workload among adults, elderly and very elderly patients in the intensive care unit. Rev Esc Enferm USP. 2009; 43(Esp 2): 1284-91.

23. Inoue KC, Kuroda CM, Matsuda LM. Nursing Activities Scores (NAS): carga de trabalho de enfermagem em UTI e fatores associados. Cienc Cuid Saude. 2011; 10(1): 134-140.

24. Coelho FUA, Queijo AF, Andolhe R, Gonçalves LA, Padilha KG. Carga de trabalho de enfermagem em unidade de terapia intensiva de cardiologia e fatores clínicos associados. Texto contexto-enferm. 2011; 20(4): 735-41.

25. Queijo AF, Martins RS, Andolhe R, Oliveira EM, Barbosa RL, Padilha KG. Nursing workload in neurological intensive care units: Cross-sectional study. Intensive Crit Care Nurs. 2013; 29(2): 112-6.

26. Carmona-Monge FJ, Jara-Pérez A, Quirós-Herranz C, Rollán-Rodríguez G, Cerrillo-González I, García-Gómez S, et al. Assessment of nursing workload in three groups of patients in a spanish ICU using the nursing activities score scale. Rev Esc Enferm USP. 2013; 47(2): 331-6.

27. Siqueira EMP, Ribeiro MD, Souza RCS, Machado FS, Diccini S. Correlação entre carga de trabalho de enfermagem e gravidade dos pacientes críticos gerais, neurológicos e cardiológicos. Esc Anna Nery. 2015; 19(2): 233-238.

28. Oliveira LB, Rodrigues ARB, Püschel VAA, Silva FA, Conceição SL, Béda LB et al. Avaliação da carga de trabalho no pósoperatório de cirurgia cardíaca segundo o Nursing Activities Score. Rev Esc Enferm USP. 2015; 49(Esp): 80-86.

29. Nogueira LS, Domingues CA, Poggetti RS, Sousa RMC. Nursing Workload in Intensive Care Unit Trauma Patients: Analysis of Associated Factors. PloS One [Internet]. 2014 [citado 30 jan 2015]; 9(11): e112125. Disponível em: https://www. ncbi.nlm.nih.gov/pubmed/25375369

30. Adomat R, Hewison A. Assessing patient category/dependence systems for determining the nurse/patient ratio in ICU and HDU: a review of approaches. J Nurs Manag. 2004; 12 (5): 299-308. 